\title{
CORTICOSTEROID THERAPY IN CURRENT DERMATOLOGICAL PRACTICE
}

\author{
H. W. ChadField, M.R.C.P.E. \\ Consultant Dermatologist, Wolverhampton, West Bromwich, Dudley and Stourbridge Groups of Hospitals
}

THE introduction of corticosteroid drugs in 1949, thanks to the pioneer work of Kendall, Reichstein and Winterstein, ranks as one of the greatest medical advances of all time and probably the greatest since antibiotics were discovered a couple of decades ago. In no other field, probably, has their application been of greater service than in dermatology, and they have now been employed long enough for a reassessment of their worth.

Under the influence of the pituitary adrenocorticotrophic hormone, which is probably under hypothalamic control, a variety of steroids is secreted by the adrenal gland including hydrocortisone (or cortisol), corticosterone, aldosterone and sex hormones like progesterone, cestrogens and androgens. There is a reciprocal relationship between the concentration of circulating corticotrophin and of adrenal cortical hormones. Hydrocortisone, which like corticosterone and cortisone belongs to the I I-oxysteroid group of adrenal corticosteroidsso called because an $=\mathrm{O}$ or $-\mathrm{OH}$ group is attached to $C$ in the II position-is secreted by the human adrenal cortex in far greater amounts than any of the other hormones. Cortisone, the first of the synthetic corticosteroids to be manufactured, is not produced by the adrenal gland, and when administered therapeutically is probably converted into hydrocortisone which is the active hormone. In recent years several hundred analogues of cortisone have been prepared by inserting additional groups into the parent structure in an attempt to eliminate undesirable mineralocorticoid sodium retaining and potassium losing effects by virtue of the lower dosage required, but the perfect analogue has yet to be discovered. The introduction of double bonds between $I$ and 2 carbon atoms in ring $A$ produced the more potent prednisone and prednisolone. The attachment of a fluorine atom to the $9 \mathrm{C}$ position yielding fludrocortisone further enhanced the anti-inflammatory effect while adding a hydroxyl or a methyl group to the latter reduced sodium retention and produced triamcinolone and dexamethasone respectively. More recent additions are betamethasone and paramethasone.

\section{Pharmacological Effects}

A thorough understanding of the metabolic effects of these agents is essential for their proper clinical use and for the minimization of undesirable side reactions.

The corticosteroids employed therapeutically are generally called glucocorticoids because of their easily observed effects on carbohydrate metabolism. They raise the blood sugar level by decreasing the utilization of glucose, by converting amino acids into glucose (gluconeogenesis) and possibly also by hindering the tubular reabsorption of glucose. Thus their effect is diabetogenic and when they are present in excess, hyperglycæmia, glycosuria and impared glucose toler. ance may result.

Their effect on protein metabolism may be catabol or anti-anabolic with interference with protein synthesis. A negative nitrogen balance results with accompanyin retardation or cessation of growth, muscle wasting thinning of the skin, defective tissue repair, strif spontaneous bruising and osteoporosis.

Lipid metabolism is affected, there being a redistraf bution of body fat with an accumulation on the fage ('moon face') and a deposition on the nape of the nees ('buffalo hump') and the abdomen at the expense of fat in the extremities as in Cushing's syndrome.

The mineralocorticoid fraction influences electrolyte and water metabolism promoting the retention of sodium and the excretion of potassium. Edemg hypertension and heart failure may ensue.

A negative calcium balance from increased fæcos excretion and an abnormal phosphate loss may leaet osteoporosis and vertebral collapse. There is sqmie evidence suggesting that adrenal corticosteroids antagonistic to Vitamin D. The blood components $\leq$ am influenced, the number of circulating eosinophils $\bar{a}$ if lymphocytes being reduced, while polycythæmia may occur.

\section{Mode of Action and Therapeutic Effects}

To many patients the corticosteroid must indeed seeñ the wonder drug of this age, but nature exacts a hig price for the apparent miracle and corticosteroids are a double-edged weapon with a power for evil as we as for good.

The corticosteroids are apparently simple substances chemically and though their action at the molecular or even cellular level remains unexplained, they are thought possibly to be co-factors essential to enzymit reactions, the catalytic action being probably at physical rather than a chemical level. The pharmace logical use of these drugs implies the employment of unphysiological doses and depends on the production of state of hypercorticalism.

Corticosteroids and corticotrophin neither cure the condition in which they are employed nor do they eradicate the cause of the disease nor repair damaged tissue. Their therapeutic effects stem from their an inflammatory and anti-allergic actions. In large amounts they suppress the manifestations of inflammatory reaf tions, relieving the hyperæmia, œdema and exudation and reducing the formation of granulation tissue. It fortunate that this suppression of signs and symptorił of disease does not appear to interfere with any of the natural self-healing processes of the illness, and in the treatment of ordinarily fatal conditions one endeavouss to keep the patient alive long enough, one hopes, enable the pathological process to burn itself ouf Manifestations of allergic reactions including delayed hypersensitivity responses such as the Arthus phy 
nomenon, the tuberculin and Frei reactions, vasculitis and serum sickness, but excepting the immediate skin reaction to injected allergen, are suppressed, the beneficial effects being effected neither by any antagonism of the pharmacological actions of histamine or of 5-hydroxytryptamine nor by any inhibition of the antigen - antibody reaction, but probably by a blockage of the effects of histamine on the tissues and by an effect on capillary permeability. The active formation of antibodies (active immunity) is suppressed, but there is no effect on injected antibodies (passive immunity).

Cutaneous effects of corticosteroid therapy include also follicular hyperkeratinization, decreased formation of sebum and increased sweating. As already stated the skin participates in the depletion of body protein, becoming atrophic and fragile and striæ may occur from thinning of collagenous and elastic fibres. Purpura may occur from collagen degeneration as in senile purpura. Dorfman and Schiller (1958) have shown that the consistence and permeability of the intercellular ground substance of connective tissue is involved, the metabolism of its mucopolysaccharide constituent being reduced. Corticotrophin may cause pigmentation (probably from contamination with the melaninstimulating hormone of the pars intermedia) and spider nævi.

\section{Hazards of Systemic Therapy}

Many of the hazards have been implicit in the consideration of the metabolic effects. The list is impressive and equalled probably by no other drug.

Side effects, or therapeutically undesirable but inevitable and unavoidable effects, of these drugs result from high dosage employed over a short period of time or more frequently, from moderate doses given over some weeks or months. They result from excess of hormone and present as varying degrees of Cushing's Syndrome. Nearly all patients receiving a dose of the order of $15 \mathrm{mg}$. of prednisolone daily will in time develop one or other of these. They include moon face, buffalo hump, striæ, œdema, hypertension, congestive cardiac failure, the unmasking of latent diabetes or the aggravation of existing diabetes, osteoporosis (including crush fracturs of the spine), psychological changes (euphoria, depression, disorientation, hypomania or suicidal tendencies), androgenic manifestations (acne, hirsutism and amenorrhœa, probably from impurities) and developmental abnormalities when administered during pregnancy.

Toxic effects are not seen from the true hormone itself but may appear following the use of the individual analogues. 'Primary myopathy' with muscle damage involving proximal muscle groups of the limbs was described by Williams (r959) following the use of triamcinolone and dexamethasone. Other possible toxic effects from triamcinolone are headache, dizziness, anorexia, loss of weight and telangiectasis of the face, trunk and limbs.

More recently evidence has been presented to support the contention that a moderate or high dose of corticosteroid therapy over a prolonged period of time may cause posterior subcapsular lens opacities (Black, Ogelsby, Von Smallman, and Bunim (1960)).

Altered tissue reactions may cause enhancement of infective processes (e.g. the aggravation of pre-existing infections like tuberculosis or, as shown by Goldstein and Rambo (1962), cryptococcosis, and the masking of symptoms and signs of infection) and delayed wound healing. Activation of peptic ulceration (aided probably by an increased secretion of hydrochloric acid, pepsinogen and trypsinogen and by a local interference with the replacement of gastric mucosa continually shed in the normal process of exfoliation) may cause perfora- tion and hæmorrhage, and perforation of the colon may occur in cases of diverticulitis. There may be a liability to vascular thrombosis and thrombo-embolic complications possibly from inhibition of fibrolysins.

Corticosteroid withdrawal. It is well known that the abrupt termination of a course of corticosteroid therapy may be followed by adrenocortical atrophy from suppression of corticotrophin production, through recent work by Treadwell, Savage, Sever and Copeman (1963) suggests that this atrophy is never complete and stresses the failure of pituitary response to subnormal levels of circulating hydrocortisone.

Abrupt cessation of treatment or the supervention of a state of relative insufficiency (as when the increased requirements of a stressful situation are not met) may also, paradoxically, produce arteritis and other manifestations of damage to connective tissue and result in a condition resembling naturally occurring polyarteritis nodosa or systemic lupus erythematosus (S.L.E.) which may be superimposed on a pre-existing collagenosis.

In one series of cases Nordin (1960) reported that death attributable to corticosteroid therapy occurred in $8 \%$ while a M.R.C. review (196r) of 107 patients with S.L.E. treated with steroid hormones over a period of at least two years showed that complications attributable to this treatment included 16 occurrences of hypertension, nine of psychotic change, five of infection, four of gastro-intestinal hæmorrhage, two each of crushed fractures of vertebræ, dyspepsia and hirsutism and one of glycosuria.

\section{Contra-indications}

Systemic corticosteroid therapy should be withheld in Cushing's syndrome, peptic ulceration, diverticulitis, psychoses, chronic nephritis and in infections insusceptible to antibotics.

It should, when required, be administered with caution in cardiovascular disease, diabetes mellitus, osteoporosis, pregnancy, thrombotic diseases and where a psychiatric case history exists.

In general, contra-indications are less likely to be binding, and benefit more likely to ensue, in the case of severe acute and short lived conditions than in persistent disorders.

\section{Routine precautions}

It follows that the taking of a careful personal family history and a complete general medical examination are pre-requisites for a decision on the employment of these drugs. The examination must include a recording of the blood pressure and weight and an analysis of the urine and must be repeated weekly while the patient remains under treatment. In addition an $\mathrm{X}$-ray must be taken of the chest to exclude active or latent tuberculosis and of the skeleton (and repeated at intervals of four months while under risk) to exclude osteoporosis, while a history of peptic ulceration demands a barium meal. Serum sodium and potassium estimations may be required and routine ophthalmological examinations have been suggested for some cases.

Steroid tablets should be chewed with a meal or, especially when there is a history of dyspepsia, be dispensed in enteric-coated form. Antacids may be required. It is customary to administer potassium supplements (e.g. I g. potassium chloride t.d.s.). When osteoporosis is present and steroid treatment is considered essential, further drain on bone calcium may be minimized by the daily administration of $3 \mathrm{~g}$. of calcium, 50,000 units of Vitamin $D$ and of an anabolic steroid (e.g. norethandrolone $30 \mathrm{mg}$ ).

A warning card giving the patient's name and address and the dosage of treatment should be carried by him 
at all times to guard against interrupted dosage and to ensure that the increased demands of any stressful situation (e.g. accident, infection or operation) are met.

\section{Dosage and Methods of Employment}

The initial dosage required to bring a condition under control may vary from as little as $15 \mathrm{mg}$. of, say, prednisolone a day in divided doses in a simple benign disorder like eczematous dermatitis to as much as $400 \mathrm{mg}$. daily in divided doses for a severe fatal condition like pemphigus vulgaris. In general, the former type of case requires a dose in the region of 30 or $40 \mathrm{mg}$. of the aforementioned drug (or equivalent dose if a different analogue is used) daily at the commencement of therapy while an average initial requirement in the latter group is $80 \mathrm{mg}$. daily. The assessment of the appropriate dose is a matter of judgement based largely on experience. Some aim at a dosage which is a little above the minimum required for suppressive action while others prefer to feel their way more cautiously with smaller doses which may have to be increased rapidly and sometimes even doubled to achieve control.

The disease having been brought under control, the dose should be gradually reduced to the amount which is necessary to keep the patient comfortable and usually to below the level required to suppress the symptoms entirely. After a further period of time an attempt at slower reduction with decrements of $5 \mathrm{mg}$. or less of this drug every three or four days is made. If control is lost the dose may have to be increased temporarily to above the level from which it was last reduced after which a further attempt at lowering it is undertaken. The secret of success in dose reduction is patience and it can sometimes be obtained by subtracting as little as $\mathrm{I} \mathrm{mg}$. from the daily dose at intervals of 3 or 4 days, achieving the desired object almost by stealth as it were. The I mg. tablets of prednisolone are particularly helpful in this respect.

The maintenance level is usually between $\mathrm{I} O$ and I $5 \mathrm{mg}$. a day but may vary between 5 and $30 \mathrm{mg}$. or more daily depending upon the severity of the condition and probably other factors as well.

The reduction of the dose to the lowest possible level and the eventual discontinuation of the drug whenever possible must always be the physician's set aim and purpose.

\section{Indications for Systemic Treatment}

It is obvious that the decision whether or not to employ corticosteroids systemically involves a careful appraisal of all relevant circumstances, considering the risks involved against the likely benefits. At all times it is a calculated risk comparable at least, in my opinion, to the hazard of a laparotomy and probably far greater. Yet, under the relentless pressure of propaganda from drug $\bar{z}$ firms, and sometimes because of the insistence of $\stackrel{\Phi}{\complement}$ the patients themselves, there is a growing tendency $c$ to use these drugs, often in inadequate dosage, for $\overrightarrow{\vec{F}}$ conditions which are either insusceptible to them $\stackrel{9}{\rightarrow}$ or which, if amenable to their action, could be treated far more safely and adequately by more $\frac{\bar{\sigma}}{\bar{\omega}}$ conventional methods. Particularly to be con- $\frac{\widehat{D}}{\widehat{D}}$ demned is their employment, sometimes in panic $\stackrel{\curvearrowright}{\circ}$ fashion, before a diagnosis has been made. The क rash is then partially or completely suppressed, $\vec{\circ}$ rendering the condition virtually undiagnosable and its management complicated, to say the least. $\vec{\sigma}$ Worse still is the suppression, not only of the rash $\frac{\sigma}{0}$ but of the vitally important information that these $\frac{0}{3}$ drugs have been used. In the words of Sulzberger $\frac{3}{\omega}$. and Wolf (1952), anyone who prescribes or administers these drugs lightly is not living up to his responsibilities as a physician.

In actual fact, the indications for systemic treat- N ment are fairly clear and there are, broadly, three o main groups of conditions which may be benefited.

\section{Severe disorders threatening life}

The collagenoses. Diseases characterized by $\frac{\vec{D}}{D}$ fibrinoid degeneration of connective tissue an $\bar{Z}$ believed to be auto-immune disorders in view of $\frac{\mathbb{}}{-}$ the presence in the serum gammaglobulin fraction $\vec{\emptyset}$ of antibodies to a large number of autologow tissue components.

Acute systemic lupus erythematosus (S.L.E.)

Dermatomyositis

Polyarteritis nodosa

Vesico-bullous diseases. The pemphigus group $\frac{2}{\circ}$ of diseases (pemphigus vulgaris, pemphigus $\stackrel{2}{\rightarrow}$ vegatans, pemphigus foliaceous and pemphigus $\frac{\vec{O}}{3}$ erythematosus), pemphigoid and benign mucous membrane pemphigoid (ocular pemphigus), severe erythema multiforme (including Stevens-Johnson syndrome).

Epidermolysis bullosa (severe dystrophic variety)

Toxic epidermal necrolysis (Lyell)

Herpes gestationes

Exfoliative erythrodermia

Severe anaphylactoid purpura

Rarer miscellaneous dermatoses

Pyoderma gangrenosum

Recurrent febrile non-suppurative panni- $N$ culitis-(Weber-Christian)

Lethal midline granuloma of the face

Wegener's respiratory granulomatosis

Certain reticuloses and reticulo-endothelioses

Certain lepromatous reactions

Severe acute self-limiting eruptions

Severe sensitization or contact dermatitis 
Severe drug eruptions

Acute widespread lichen planus (including lichen planus bullosus)

Severe angioneurotic œdema with laryngeal involvement

Acute radio-dermatitis

Severe exacerbations of chronic eczema (e.g. in atopic eczema)

Systemic Lupus Erythematosus. There is general agreement that steroids should be reserved for seriously ill patients and not used in the benign phases of this condition. Initial doses of the order of 50 or $60 \mathrm{mg}$ of prednisolone daily are likely to be required and may have to be increased should a rapid therapeutic response not occur. Doses of up to $400 \mathrm{mg}$ daily of this drug may, on occasion, be necessary in case of a crisis. Under the influence of corticosteroid therapy, subjective improvement occurs in the first week and objective findings like pleural and pericardial effusions clear shortly afterwards, though cutaneous lesions are slower to resolve, taking up to a month. Unfortunately, renal disease remains unaffected. Objective findings like leucopenia, hyperglobulinæmia and elevation of the ESR tend to improve though they by no means invariably return to normal and the ESR is not a reliable guide to dosage. The characteristic L.E. cells, formed by the phagocytosis by polymorphonuclear leucocytes of extruded degenerate nuclear material of other polymorphs with the production of swollen cells containing a large basophilic amorphous inclusion body and an eccentric nucleus, likewise tend to become fewer, but rarely disappear completely.

Once a remission has been induced the drug should be gradually discontinued if possible, or reduced to a suitable maintenance level below which signs of activity begin to appear. The dosage may thereafter have to be increased in case of a relapse.

Despite the effectiveness of steroids in this condition it is, as yet, uncertain how far the eventual outcome is influenced and the prognosis remains grave.

Dermatomyositis. This condition belongs to the category of polymyositis, a composite group of degenerative muscular disorders characterized by muscular weakness or paralysis and sometimes symptomatic of a visceral or other neoplasm.

Some cases, especially of the juvenile type, are responsive to steroids and the requirements approximate those of an average case of S.L.E. though a particularly careful watch must be maintained on the nitrogen balance because of the considerable breakdown of muscle protein in this disease.

Polyarteritis Nodosa. The cutaneous mani- festations of this condition may be eruptions of scarlatiniform, urticarial, vesicular or bullous character or ulcerations. Prednisolone in a dose of 60 to $100 \mathrm{mg}$ daily may be required initially and a prolonged maintenance dosage is usually necessary. There is some danger that rapid healing of the lesions may be accompanied by arterial occlusion.

Progressive Systemic Sclerosis (Diffuse Scleroderma) is not usually an indication for systemic corticosteroid therapy in view of the reported supervention of malignant hypertension and uræmia in cases so treated.

The Pemphigus group of diseases (particularly pemphigus vulgaris) and the less lethal conditions of pemphigoid and benign mucous membrane pemphigoid (ocular pemphigus) are greatly benefited by systemic steroid treatment with general constitutional improvement and a diminution in the rate of bulla formation within forty-eight hours and a cessation of their appearance usually within a week. A relatively high dosage of usually at least $60 \mathrm{mg}$ of prednisolone daily, and sometimes considerably more, is required initially in the pemphigus group and must be rapidly doubled if ineffective to prevent loss of control.

Recently Lever and White (1963) stress the importance of early treatment with initial high doses and advocate a daily dose of 24 tablets of $5 \mathrm{mg}$. of prednisolone for mild cases and 36 tablets of the drug for cases showing more extensive involvement with increase of dosage to 36 or 48 tablets respectively should new lesions continue to appear after 5 days. The requisite dosage is continued for 6 to 8 weeks until healing is complete and then reduced in stages over a period of some months to the lowest possible maintenance dose.

In one series of cases the mortality with steroids, though still considerable, was reduced to one third of what it was in the pre-steroid era. Pemphigoid is more easily controlled and smaller doses, e.g. $40 \mathrm{mg}$ daily initially, may be effective.

Erythema Multiforme. This is a syndrome due to multiple causes (e.g. drugs, focal bacterial infection, viruses, reticuloses, etc.) and an attempt to establish a concise ætiological diagnosis is essential before a therapeutic decision is made. Steroids may be required in the severe form associated with mucosal (e.g. conjunctival, oral, genital) lesions, and. known as the StevensJohnson syndrome.

Behcet's Triple-Symptom Complex of recurrent genital and oral ulceration with associated eye lesions and at times erythema-multiforme-like eruptions may be benefited.

Epidermolysis Bullosa. A hereditary and familial condition characterized by a vulnerability of 
the skin to trauma with the formation of vesicles and bullae, usually over bony prominences of the extremities. Systemic corticosteroids may be of value in the severe dystrophic and lethalis forms of the disease which may involve the oropharyngeal and œsophageal mucosæ with resultant bulla formation, ulceration, scarring and sometimes stenosis of the upper alimentary tract.

Toxic Epidermal Necrolysis (Lyell). This acute fulminating febrile disturbance which appears as an inflammation of mucous membranes and a rapidly spreading erythema with ensuing blistering, loosening and shedding of the superficial layers of the skin, produces a characteristic scalded appearance and is frequently a manifestation of drug intoxication (as from phenolphthalein, phenylbutazone or gold) and may respond to corticosteroid therapy.

Herpes Gestationes. Steroids may on occasion be justified in this variant of dermatitis herpetiformis which is associated with pregnancy.

Exfoliative Dermatitis, whether primary, complicating a pre-existing dermatosis (like eczema, psoriasis, seborrhœic dermatitis or lichen planus), a form of drug reaction or a manifestation of a reticulosis may require the hormone systemically.

Pyoderma Gangrenosum. This sometimes alarmingly progressive ulcerative condition of the skin which is sometimes associated with ulcerative colitis is, in my experience, frequently responsive to systemic steroid therapy.

Weber-Christian disease is a recurrent febrile non-suppurative nodular inflammatory disorder of adipose tissue involving the subcutaneous and, on occasion, also the omental, mesenteric and other adipose tissues with subsequent fat necrosis. Steroids may be of value (though a fatal case with visceral involvement of which I have personal knowledge, did not respond) and hence it has been suggested that this may be a collagen adisese.

\section{Lethal Midline Granuloma of the face,} characterized by a progressive destruction of the centre of the face with ulceration and necrosis and the allied Wegener's respiratory granulomatosis, a necrotizing vasculitis of the lungs and skin with glomerulonephritis (which may be a variant of polyarteritis nodosa) are indications for the use of systemic corticosteroid therapy.

The Reticuloses. Oral steroids may relieve some of the cutaneous manifestations of acute and chronic leukæmia, of Hodgkins' disease, of mycosis fungoides, and of allied conditions in virtue of their anti-inflammatory and anti-puritic effects.

Certain reticulo-endothelioses like LettererSiwe disease of infancy may be benefited.
In Severe Sensitization and Contact Dermatitis the cause must always be sought and, once $\mathbb{D}$ removed, the course of the illness may be consider- $c$ ably curtailed by the systemically administered hormone.

In Drug Reactions steroids should be reserved for severe cases only, when other methods of treatment have failed.

When the patient has become sensitive to a $\stackrel{\square}{\square}$ vitally needed drug its continued administration क may, on occasion, be rendered possible, or de- $\vec{A}$ sensitization attempted, by concomitant steroid therapy, though these procedures are not without risk.

Acute Radiodermatitis. In a recent series (Sweet 1962) of accidentally produced superficial $\stackrel{3}{3}$. $\mathrm{X}$-ray burns, it was felt that though treatment with large doses of oral prednisolone had no effect in preventing epidermal loss with epilation, of it postponed the subsequent necrosis of the dermal on and deeper tissues (probably by inhibiting the vascular occlusion resulting from fibrosis of the walls of blood vessels) and appeared to lessen pain and help the maintenance of function.

Atopic eczema calls for special comment. In $\frac{\mathbb{D}}{0}$ a very chronic disorder of this nature the patients 3 have, of necessity, learned to live with they $\frac{\Phi}{1}$ disability and this they are prepared to do. When however, the magic drug capable of causing relief o in almost miraculous fashion is exhibited an then subsequently withdrawn, they frequent $\overrightarrow{f y}$ become intensely depressed, intolerant of the slightest recrudescence and unable to adjust themselves to the new state of affairs. I have found

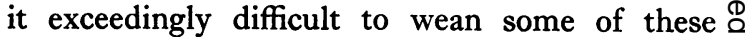
patients from their steroids once effective relief $\overrightarrow{\vec{P}}$ has been obtained and to this extent the drug may 3 be regarded as one of addiction. To borrow a phrase-'We have got a tiger by the tail and we? dare not let go'-for on every occasion on which an effort is made to diminish the dosage the patient's skin flares up violently. Nevertheless, some cases of atopic eczema are so severe and disabling and interfere with all the patients activities to such an extent that systemic corticosteroid therapy may become permissible, but 웅 one must be prepared to continue minimal suppressive therapy for months or even years if necessary.

Systemic corticosteroids are not advised in infantile eczema nor in psoriasis (except the erythrodermic type) nor in alopecia areata.

\section{Local Corticosteroid Therapy}

Corticosteroid preparations for topical therapy are now in very wide use and the multiplicity? of available preparations equals that of the systemically used drugs and is bewildering.

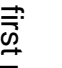


Hydrocortisone was the first of these to be employed, cortisone and corticotrophin being ineffective topically. Other preparations now contain prednisolone, methyl prednisolone, triamcinolone, dexamethasone, betamethasone or fluorocortisone. It was found that a combination of 16-alpha and 17-alpha hydroxyl groups of triamcinolone with acetone to form the acetonide, greatly increased topical activity. Two new synthetic fluorinated corticosteroids for topical use, fluocinolone (Synalar, I.C.I.) and flurandrenolone (Drenison, Lilly) contain the same acetonide group and are claimed to have greater antiinflammatory activity.

These preparations vary in strength from $0.025 \%$ to $2.5 \%$, largely according to their individual potency. Most of them are available as lotions, creams and ointments and some of them as ærosol sprays. Recently, one of these preparations has become available in emollient dental paste (Adcortyl A in Orobase, Squibb) for the treatment of acute and chronic oral lesions.

Properly used, these preparations are of inestimable value in the treatment of cutaneous diseases, but there is a growing tendency to use them indiscriminately as a first line of attack in unsuitable cases, sometimes undiagnosed, or in conditions which can be treated more effectively and cheaply by other means. Again, it must be stressed that this treatment, as in the systemic variety, is suppressive only and does not cure, and must be preceded by adequate diagnosis and an attempt to find the cause of the illness. Though cases of herpes simplex can be seriously aggravated by such treatment, little harm, fortunately, results as a rule in unsuitable casesexcept to the exchequer, for these drugs are still very costly. With the exception of fluorocortisone, systemic absorption of these steroids, administered topically, is believed to occur in minute amounts only, though the new occlusive techniques are likely to facilitate this process.

As in the case of dermatological preparations generally, the base is no less important than the active ingredient and differences in composition probably account for varying therapeutic response and tolerance, sensitization to the corticosteroid being virtually unknown. As a general rule, sprays, lotions and creams are indicated for acute and sub-acute lesions and ointments for chronic conditions.

Corticosteroids applied locally are pleasant to use, do not soil clothing and are effective in virtue of their anti-inflammatory and antipruritic effects (they are known to cause vasoconstriction when applied to healthy unbroken skin). Their effectiveness is limited by the thickness of the horny layer of skin and conse- quently they are more beneficial when applied where the epidermis is thin (e.g. on the eyelids, face and neck) than on such areas as the palms and soles. They should be applied sparingly and rubbed gently into the affected areas three or four times daily. They should not be discontinued abruptly when the condition improves, but applied at longer intervals. Covering dressings are usually unnecessary unless there be friction from clothing.

Almost every known topical corticosteroid preparation is now combined with at least one other active, usually antibacterial, substance. Provided the indications exist, e.g. a microbic or fungal infection complicating eczema, there is no harm and, indeed, benefit to be derived from the combination, but the use of multiple preparations in 'blunderbuss' fashion (as when steroid, antibiotic and potentially sensitizing local anæsthetic preparation are combined) is not advised. I have, however, found one corticosteroidantibiotic-pancreatic enzymic combination (Chymar ointment, Armour) useful in necrotic slough-covered ulcerative conditions. Other substances usefully combined with corticosteroids are dequanilium chloride (Dequalone P.-A. \& H.), domiphen bromide (Ultracortenol with Bradosol cream, Ciba), iodochlorhydroxyquinoline (Vioform, Ciba) and soframycin with gramicidin (Sofradex, Roussel) the latter being useful in otitis externa.

\section{Indications}

Topical use of corticosteroid preparations is often effective in the following conditions:-

Eczema and dermatitis, including atopic eczema, infantile eczema, contact dermatitis, neurodermatitis

Intertriginous eruptions, e.g. seborrhœic, involving the axillæ, groins and retro-auricular spaces (including otitis externa)

Ano-genital pruritus

Facial and flexural psoriasis

Chronic discoid lupus erythematosus-preliminary impression seems to indicate that one of the latest preparations (Synalar, in ointment form) may be of great value in this condition. If this can be substantiated it would indeed represent a great advance in the treatment of this cosmetically baneful condition which has hitherto necessitated the use of ophthalmologically dangerous drugs

Lichen sclerosus and atrophicus (some cases)

Aerosols are not, in general, favoured by most authorities, largely on the grounds of expense, but I have found one corticosteroid-antibiotic preparation in this form (dexamethasone with neomycin-Decaspray, Merck, Sharp \& Dohme) 
very useful in infected exudative eczematous conditions.

\section{Plastic Occlusive Dressings}

These have recently been introduced in this country by Overton (1962) following the example set in the United States by Sulzberger and Witten (196r) and by Scholtz (196r) who adapted the method used by Gärb (1960) in treating hyperkeratotic nævi with podophyllin. The corticosteroid cream (e.g. Synalar or Drenisonsometimes mixed, for the sake of economy in widespread lesions, with up to ten times its quantity of ung. aquosum) is applied to the affected areas and covered with thin flexible plastic material such as polythene which is secured and made airtight with cellophane tape. An overlying tubular gauze dressing may be applied. Hall-Smith (1962) has recently reported on the employment of this technique in seventytwo patients suffering from chronic and recalcitrant dermatoses including psoriasis, chronic fissured eczema and pompholyx of the hands, atopic dermatitis, lichen simplex chronicus, pustular bacteride of the soles, hypertrophic lichen planus, keratosis pilaris and icthyosis simplex. Most of the cases showed rapid improvement though there was a tendency to relapse when the treatment was discontinued. This treatment has also been used in localized scleroderma (morphœa) involving a limb. The dressings are changed every fortyeight hours or more often, depending upon the tolerance of the patient.

The rationale of this method of treatment is the assurance of more intimate contact of the medicament with the lesion and enhanced percutaneous absorption resulting from the increased local sweating and the promotion of epidermal maceration.

Undesirable effects must be guarded against. These include skin infections (folliculitis, boils or abscesses), danger from fire and possible impaired thermal homeostasis.

\section{Occlusive Hydrocortisone Bandaging}

This addition to the conventional range of occlusive bandages is a more recent development still. Holti and Ingram (1963) recommend the use of cotton bandages impregnated with hydrocortisone in a silicone barrier cream (Smith and Nephew) which can be left in place (covered with e.g. tubular gauze bandages) it is claimed, for up to six weeks. It is apparently particularly useful in cases of infantile eczema.

\section{Mucosal Application of Corticosteroids}

Triamcinolone acetonide (Adcortyl A) in orobase (a new type of adhesive vehicle composed ô gelatin, pectin and carboxymethyl cellulose a liquid petrolatum polyethylene base intended $\mathrm{t}$ maintain medication at the site of application in the mouth for prolonged periods) has recenthy been introduced for the treatment of recurreft ulcerative stomatitis, erosive lichen planus, dentuge dermatitis, aphthous stomatitis, black hairy tongu $\overline{\overline{0}}$, mucosal erythema multiforme and mucosal lupos erythematosus. It is advised that a thin coating, $\frac{1}{4}$ inch or less, be applied to the lesion two or three times daily after meals.

\section{Intralesional Therapy}

A fine suspension of corticosteroid (e.g. triangcinolone acetonide) is injected slowly into the superficial layers of the dermis in usually 0.1 r amounts at sites $\mathrm{I} \mathrm{cm}$. apart at weekly intervalof This form of treatment has been recommendẹd for the following conditions:

Lichen simplex chronicus

Cysts of acne vulgaris

Localised psoriasis

Chronic discoid lupus erythematosus however, under Local Application)

Granuloma annulare

Sarcoid

Hypertrophic lichen planus

Necrobiosis lipoidica diabeticorum

Pretibial myxœedema

Penile plaques of Peyronie's disease

Nail dystrophies (from psoriasis, lichen planu etc.) (Gerstein, 1962)

Hæmorrhagic lichen sclerosus et atrophic (Pillsbury and Shelley, 1963).

Unfortunately, the effects tend to be tempora $\overrightarrow{\overline{6}}$ and dermal atrophy, and occasionally ulceration, may occur. Some systemic effect may be exerte and therefore due care must be taken in the selection of cases.

\section{Corticotrophin}

Pituitary corticotrophin, a polypeptide co: sisting of 39 amino-acids linked together, can only be administered by injection and has now largely been replaced by the orally effective corticosteroids which possess obvious advantage.

The therapeutic and side effects are very largely the same, but it produces a clinical response in a shorter time than an equivalent amount corticosteroid and the effects wear off less rapidus than those of cortisone when the drug is discow tinued. Cortisone can be regulated more accuratedy than corticotrophin, the effects of which are monte liable to fluctuation and presuppose a normalfy responsive adrenal cortex.

For temporary maximal stimulation 60 units $\overrightarrow{\phi_{f}}$ 
Acthar gel 12 hourly (for not more than 2 days) and for moderate stimulation 40 units can be given.

For practical purposes the use of corticotrophin is largely confined to initiating a therapeutic response and to activating the adrenal cortex prior to discontinuing steroid therapy. In the latter case 20-40 units of Acthar gel by intramuscular injection twice weekly for three to four weeks may be given.

In some cases of severe life-threatening derma- toses where a state of apparent refractoriness to even very large doses of corticosteroids appears to have been reached, their partial substitution or supplementation by appropriately spaced injections of corticotrophin sometimes appears to be of value.

It is evident that though the introduction of adrenal corticosteroids has brought an addition of the first magnitude to the dermatological armamentarium, it has in no way over-simplified the management of diseases of the skin.

\section{REFERENCES}

Black, R. L., Ogelsby, R. B., Von Smallman, L., and Bunim, J. J. (1960): Posterior Subcapsular Cataracts induced by Corticosteroids in Patients with Rheumatoid Arthritis, $\mathcal{F}$. Amer. med. Ass., 174, 166.

Dorfman, A., and Schiller, S. (1958): Effects of Hormones on the Metabolism of Acid Mucopolysaccharides of Connective Tissue, Rec. Progr. Hormone Res., 14, 427.

Garb, J. (1960): Nevus Verrucosus Unilateris Cured with Podophyllin Ointment, Arch. Derm., 81, 606.

Gerstein, W. (1962): Psoriasis and Lichen Planus of Nails: Treatment with Triamcinolone, Ibid., 86, 79.

Goldstein, E., and Rambo, O. N. (1962): Cryptococcal Infection Following Steroid Therapy, Ann. intern. Med., 56, 114 .

Hall-Smith, S. P. (1962): 'Polythene' Coverings in Dermatological Treatment, Brit. med. F., ii, 1233.

Holti, G., and INGRAM, J. T. (1963): Physiotherapy in Dermatology. Lancet, i, 14I.

Lever, W. F., and WhITE, H. (1963): Treatment of Pemphigus with Corticosteroids, Arch. Derm., 87, 12-26.

M.R.C. Collagen Diseases and Hypersensitivity Panel (1961): Treatment of Systemic Lupus Erythematosus with Steroids, Brit. med. F., ii, 915.

Nordin, B. E. C. (1960): Side Effects of Systemic Adrenal Steroid Therapy, Brit. F. Derm., 72, 40.

Overton, J. (1962): Lecture to Consultants Course, St. John's Hospital for Diseases of the Skin, London.

Pillsbury, D. M., and Shelley, W. B. (1963): Hæmorrhagic Mehen Sclerosis et Atropicus. Arch. Derm., 87, 338.

Scholz, J. R. (196I): Topical Therapy of Psoriasis with Fluocinolone Acetonide, Ibid., 84, 1029.

SulzBERGER, M. B., and Wolf, J. (1952): 'Dermatology: Essentials of Diagnosis and Treatment', Third Edition, p. 306. Chicago: Yearbook Publishers Inc.

-, and Witten, V. H. (1961): Thin pliable plastic films in topical dermatologic therapy, Arch. Derm., 84, 1027.

SweEt, R. D. (1962): Acute Accidental Superficial X-Ray Burns, Brit. F. Derm., 74, 392.

Treadwell, B. L. J., Savage, O., Sever, E. D., and Copeman, W. S. C. (1963): Pituitary-Adrenal Function During Corticosteroid Therapy, Lancet, $\mathbf{i}, 355$.

Williams, R. S. (I959): Triamcinolone Myopathy, Ibid., i, 698. 